\title{
On the neutralino as dark matter candidate. II. Direct detection
}

\author{
A. Bottino, V. de Alfaro, N. Fornengo, G. Mignola and S. Scopel \\ Dipartimento di Fisica Teorica dell'Uniuersità di Torino and INFN, Sezione di Torino, Via P. Giuria 1, 10125 Turin, Italy
}

Received 31 August 1993

\begin{abstract}
Evaluations of the event rates relevant to direct search for dark matter neutralino are presented for a wide range of neutralino masses and for various detector materials of preeminent interest. Differential and total rates are appropriately weighted over the local neutralino density expected on theoretical grounds.
\end{abstract}

\section{Introduction}

The most natural way of searching for neutralino dark matter is provided by direct experiments where the effects induced in appropriate detectors by neutralino-nucleus elastic scattering may be measured. The aim of this paper is to present the evaluation of the event rates relevant to these direct measurements and to discuss the experimental sensitivities required in this search for it to be successful.

Our present analysis refers to a wide range for the neutralino masses, $20 \mathrm{GeV} \leq m_{x} \leq 1 \mathrm{TeV}$, and examines various detector materials of present experimental interest: $\mathrm{Ge}, \mathrm{NaI}, \mathrm{Xe}, \mathrm{CaF}_{2}$. The theoretical framework is provided by the Minimal SuSy Standard Model (MSSM), implemented with a general GUT assumption, as discussed in the companion paper [1] (this reference will hereafter be referred to as paper I). The model depends on a number of frec parameters which, apart from the masses of a few particles which come into play, are chosen here to be $M_{2}$, $\mu, \tan \beta$. The definition of these parameters is recalled in paper $I$.

Correspondence to: A. Bottino, Dipartimento di Fisica Teorica dell'Università di Torino and INFN, Sezione di Torino, Via P. Giuria 1, 10125 Turin, Italy.
In the following sections the differential and the integrated rates will be presented and discussed also in connection with the properties of the neutralino relic density.

\section{Event rates}

For the nuclear recoil spectrum we use the expression

$$
\frac{\mathrm{d} R}{\mathrm{~d} E_{\mathrm{R}}}=\sum_{i} \frac{R_{0, i}}{\left\langle E_{\mathrm{R}}^{\max }\right\rangle} F_{i}^{2}\left(E_{\mathrm{R}}\right) I\left(E_{\mathrm{R}}\right)
$$

where

$R_{0, i}=N_{\mathrm{T}} \frac{\rho_{\chi}}{m_{\chi}} \sigma_{i}\langle v\rangle$.

The various quantities in eqs. (2.1), (2.2) are defined as follows: $E_{\mathrm{R}}$ is the nuclear recoil energy, i.e. $E_{\mathrm{R}}=m_{\mathrm{red}}^{2} v^{2}\left(1-\cos \theta^{*}\right) / m_{N}\left(\theta^{*}\right.$ is the scattering angle defined in the neutralino-nucleus center of mass frame, $m_{N}$ is the nucleus mass, $m_{\text {red }}$ is the neutralino-nucleus reduced mass and $v$ is the relative velocity), $E_{\mathrm{R}}^{\max }=2 \mathrm{~m}_{\mathrm{red}}^{2} v^{2} / m_{N}$, $F\left(E_{\mathrm{R}}\right)$ denotes the nuclear form factor, $\sigma$ is the neutralino-nucleus cross section. The subscript $i$ refers to the two cases of coherent and spin-dependent effective interactions. $N_{\mathrm{T}}$ is the number density of the target nuclei and $\rho_{x}$ is the local 
neutralino matter density. Finally, $I\left(E_{\mathrm{R}}\right)$ is given by

$I\left(E_{\mathrm{R}}\right)=\frac{\left\langle v^{2}\right\rangle}{\langle v\rangle} \int_{v_{\text {min }}\left(E_{\mathrm{R}}\right)}^{l_{\text {max }}} \mathrm{d} v \frac{f(v)}{v}$.

In eq. (2.3) $f(v)$ is the velocity distribution of neutralinos in the Galaxy, measured in the Earth rest frame, $v_{\text {min }}\left(E_{\mathrm{R}}\right)$ is given by $v_{\text {min }}\left(E_{\mathrm{R}}\right)=$ $\left(m_{N} E_{\mathrm{R}} /\left(2 m_{\mathrm{red}}^{2}\right)\right)^{1 / 2}$. The averages appearing in eqs. (2.2), (2.3) denote averages over the velocity distribution in the Earth rest frame. An explicit formula for $I\left(E_{\mathrm{R}}\right)$ in the case of a maxwellian velocity distribution may be found in ref. [2].

The differential rates that will be presented in section 6 will be expressed in terms of the electron-equivalent energy $E_{\mathrm{cc}}$ rather than in terms of $E_{\mathrm{R}}$. These two variables are simply proportional: $E_{\mathrm{ec}}=Q E_{\mathrm{R}}$, where $Q$ is defined as quenching factor.

In section 6 we will also discuss the total rates, obtained by integrating $\mathrm{d} R / \mathrm{d} E_{\mathrm{ee}}$ over appropriate ranges of $E_{\mathrm{cc}}$ above the experimental energy threshold.

\section{Neutralino-nucleus elastic cross sections}

The total cross sections for neutralino-nucleus elastic scattering have been evaluated following the procedure discussed in refs. [2,3].

Here we only summarize some of the main properties. Neutralino-quark scattering is described by the amplitudes with Higgs boson exchanges and $Z$ boson exchange in the $t$-channel and by the amplitudes with squark exchanges in the $s$ - and $u$-channels. The neutral Higgs bosons considered here are the two $C P$-even bosons: $h$, $H$ (of masses $m_{h}, m_{H}$ with $m_{H}>m_{h}$ ) and the $C P$-odd one: $A$ (of mass $m_{A}$ ).

The relevant properties for these amplitudes are: (1) Higgs boson exchanges contribute a coherent cross section which is only vanishing when there is no zino-higgsino mixture in the neutralino composition [4]; (2) $Z$ boson exchange provides a spin-dependent cross section which takes contribution only from the higgsino components of $x$; (3) squark exchanges contribute a coherent cross section (due to zino-higgsino mixing) as well as a spin-dependent cross section (due mainly to the gaugino components of $\chi$ ) [5].

In the evaluations of the cross sections presented here, we have adopted for the masses of the Higgs bosons and of the sfermions the various sets of values indicated in paper I. In particular we have relaxed here the assumption, used in previous papers [2,3], that $m_{\tilde{f}}>150 \mathrm{GeV}$, since this stringent lower bound, previously reported by the CDF Collaboration [6], is significantly weakened by more refined analyses $[7,8]$. Thus the following two cases have been considered for $m_{\bar{f}}$ : (1) $m_{f}=1.2 m_{\chi}$, when $m_{\chi}>45 \mathrm{GeV}, m_{\bar{f}}=45$ $\mathrm{GeV}$ otherwise, except for the mass of the top scalar partners (the only one relevant to radiative corrections) which has been taken $\tilde{m}=3 \mathrm{TeV}$ (hereafter this choice for $m_{\tilde{f}}$ will simply be referred to as: $m_{\bar{f}}=1.2 m_{\chi}$ ); (2) $m_{\bar{f}}=3 \mathrm{TeV}$ for all sfermions. The Higgs boson mass $m_{h}$ (taken here as a free parameter) is set equal to values close to its experimental lower bound $\sim 50 \mathrm{GeV}$ [9].

Let us recall that, whereas the coherent cross section is proportional to the square of the mass number $A$ of the nucleus, the non-coherent cross section brings a spin-dependence that is commonly represented by a factor $\lambda^{2} J(J+1)$. The valucs used here for this last quantity are taken from [10]; other evaluations may be found in ref. [11].

It is worth noticing that, in the model we are considering here, the event rates for neutralino detection are largely dominated by coherent effects in most regions of the parameter space. In the small domains where spin-dependent effects dominate over the coherent ones the total rates are usually too low to allow detection. Then in the present paper we do not consider explicitly materials enriched in high-spin isotopes, even if these materials are very interesting for a search of hypothetical dark matter particles which interact with matter via substantial spin-dependent interactions. In particular for $\mathrm{Ge}$ and $\mathrm{Xe}$ we consider only natural isotopic compositions (enrichment in some isotope would not appreciably modify our results). 


\section{Nuclear form factors}

Let us turn now to the $E_{\mathrm{R}}$-dependence introduced in the nuclear recoil spectrum by the form factors. These form factors depend sensitively on the nature of the effective interaction involved in the neutralino-nucleus scattering. For the coherent case, we simply employ the standard parametrization [12]

$F\left(E_{\mathrm{R}}\right)=\frac{3 j_{1}\left(q r_{0}\right)}{q r_{0}} \exp \left(-\frac{1}{2} s^{2} q^{2}\right)$

where $q^{2} \equiv|q|^{2}=2 m_{N} E_{\mathrm{R}}$ is the squared threemomentum transfer, $s \simeq 1 \mathrm{fm}$ is the thickness parameter for the nucleus surface, $r_{0}=\left(r^{2}-\right.$ $\left.5 s^{2}\right)^{1 / 2}, r=1.2 A^{1 / 3}$ fm and $j_{1}\left(q r_{0}\right)$ is the spherical Bessel function of index 1 .

The form factor of eq. (4.1) introduces substantial suppression in the recoil spectrum unless $q r_{0} \ll 1$. A noticeable reduction in $\mathrm{d} R / \mathrm{d} E_{\mathrm{R}}$ may already occur at threshold $E_{\mathrm{R}}=E_{\mathrm{R}}^{\text {th }}=E_{\mathrm{ee}}^{\text {th }} / Q$ when $r_{0} \sqrt{2 m_{N} E_{\mathrm{R}}^{\text {th }}}$ is not small compared to unity. The actual occurrence of this feature depends on a few parameters of the detector material: nuclear radius, quenching factor, threshold energy $E_{\mathrm{ce}}^{\text {th }}$. The values of these parameters for the nuclei considered in this paper are reported in table 1 [13]. Also the values of $F^{2}\left(E_{\mathrm{ec}}^{\mathrm{th}}\right)$ calculated from eq. (4.1) are given in this table. We see that the effect of the form factor is very moderate for ${ }^{19} \mathrm{~F}$, ${ }^{23} \mathrm{Na}$ and ${ }^{76} \mathrm{Ge}$. In ${ }^{76} \mathrm{Ge}$, as compared to ${ }^{19} \mathrm{~F}$ and

Table 1

Quenching factors $Q$, threshold energies $E_{\mathrm{cc}}^{\text {th }}$, squared values of the form factor (4.1) at the threshold $F^{2}\left(E_{\mathrm{R}}^{\text {th }}\right)$ and the values $E_{\mathrm{cc}}^{0}$ where the first zero of eq. (4.1) occurs are listed for the nuclei considered in our analysis. [The values of $Q$ and $E_{\mathrm{ce}}^{\text {th }}$ are taken from refs. $[14,15](\mathrm{Ge})$, refs. [16,2] (NaI), ref. [17] $(\mathrm{Xe})$ and ref. [13] $\left(\mathrm{CaF}_{2}\right)$.]

\begin{tabular}{lllll}
\hline Nucleus & $Q$ & $E_{\text {ee }}^{\text {th }}(\mathrm{keV})$ & $F^{2}\left(E_{\mathrm{R}}^{\text {th }}\right)$ & $E_{\mathrm{cc}}^{0}(\mathrm{keV})$ \\
\hline${ }^{19} \mathrm{~F}$ & 0.09 & 8.5 & 0.84 & 378 \\
${ }^{23} \mathrm{Na}$ & 0.23 & 4 & 0.96 & 630 \\
${ }^{411} \mathrm{Ca}$ & 0.07 & 8.5 & 0.44 & 62 \\
${ }^{76} \mathrm{Ge}$ & 0.25 & 2 & 0.86 & 61 \\
${ }^{127} \mathrm{I}$ & 0.07 & 4 & 0.05 & 7.4 \\
${ }^{131} \mathrm{Xe}$ & 0.80 & 20 & 0.28 & 80 \\
\hline
\end{tabular}

${ }^{23} \mathrm{Na}$, this occurs since the larger nuclear extension is compensated by a smaller value of $E_{\mathrm{ee}}^{\text {th }}$ and a larger $Q$. The effect of the form factor on the shape of the differential spectrum as a function of $E_{\mathrm{ee}}$ depends mainly on how close to the threshold energy $E_{\mathrm{ee}}^{\text {th }}$ the first zero of the function $j_{1}\left(q r_{0}\right) / q r_{0}$ occurs (this zero is located at $E_{\mathrm{cc}}^{0} \simeq$ $10 Q / m_{N} r_{0}^{2}$. Comparing the values of $E_{\text {ee }}^{\text {th }}$ and of $E_{\mathrm{ee}}^{0}$ reported in table 1 , we see that only for ${ }^{127} \mathrm{I}$ (and marginally for ${ }^{131} \mathrm{Xe}$ ) this effect is expected to be noticeable.

In general for the spin-dependent case there are no analytic expressions for the form factors. However, numerical analyses have been performed on ${ }^{131} \mathrm{Xe}[12],{ }^{93} \mathrm{Nb}[18]$ and ${ }^{73} \mathrm{Ge}[19]$. The general feature is that these form factors have a much milder dependence on $E_{\mathrm{R}}$ as compared to the coherent ones, because only a few nucleons participate in the neutralino-nucleus scattering in this case. In our evaluations we use the results of refs. [12,19] for ${ }^{131} \mathrm{Xe}$ and ${ }^{73} \mathrm{Ge}$. For ${ }^{127}$ I we assume that the form factor has the same behaviour as in the case of ${ }^{131} \mathrm{Xe}$. For ${ }^{23} \mathrm{Na}$ and ${ }^{19} \mathrm{~F}$ we employ the analytic expression (4.1), since for these light nuclei the suppression due to nuclear size is small anyway.

\section{Neutralino local density}

As for the value of the local neutralino density, $\rho_{x}$, to be used in the rate of eq. (2.2), for any point of the model parameter space we take into account the relevant value of the neutralino relic abundance. When $\Omega_{\chi} h^{2}$ is larger than a minimal $\left(\Omega h^{2}\right)_{\min }$ consistent with the standard value for the local dark matter density $\rho_{\ell}=0.3 \mathrm{GeV} \mathrm{cm}^{-3}$, then we simply put $\rho_{\chi}=\rho_{\ell}$. When $\Omega_{\chi} h^{2}$ turns our to be less than $\left(\Omega h^{2}\right)_{\min }$, we take

$\rho_{\chi}=\rho_{\ell} \Omega_{\chi} h^{2} /\left(\Omega h^{2}\right)_{\min } \equiv \rho_{\ell} \xi$.

Here $\left(\Omega h^{2}\right)_{\min }$ is set equal to 0.05 . The effect of this procedure of rescaling the local dark matter density according to the actual neutralino relic abundance is manifest in some characteristic features of our results, as is shown in the next section. 


\section{Results}

\subsection{Germanium}

We start the presentation of our results by discussing the event rates expected for Ge-detectors *. In fig. 1 we report the integrated rate

$$
\begin{aligned}
& R\left(2 \mathrm{KeV}<E_{\mathrm{ee}}<4 \mathrm{KeV}\right) \\
& \quad=\int_{2 \mathrm{KeV}}^{4 \mathrm{KeV}} \mathrm{d} E_{\mathrm{ec}} \mathrm{d} R / \mathrm{d} E_{\text {ec }}
\end{aligned}
$$

at the representative point: $\tan \beta=20, m_{h}=50$ $\mathrm{GeV}, m_{f}=1.2 m_{\chi}$, in the form of a scatter plot obtained by varying the $M_{2}, \mu$ parameters in the ranges: $20 \mathrm{GeV} \leq M_{2} \leq 6 \mathrm{TeV}$ and $20 \mathrm{GeV}$ $\leq|\mu| \leq 3 \mathrm{TeV}$. The range of the neutralino mass $m_{\chi}$ considered here is $20 \mathrm{GeV} \leq m_{x} \leq 1 \mathrm{TeV}$; however, to simplify the presentation of our results, in fig. 1 and in the following plots for $R$, only the range $20 \mathrm{GeV} \leq m_{\chi} \leq 300 \mathrm{GeV}$ is shown

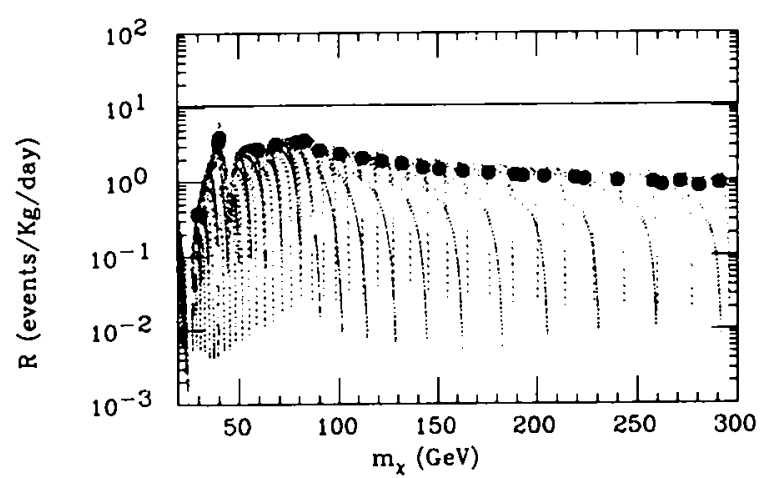

Fig. 1. Scatter plot of the integrated rate $R\left(2 \mathrm{KeV} \leq E_{\mathrm{cc}} \leq 4\right.$ $\mathrm{KeV}$ ) for neutralino-Ge interaction as a function of neutralino mass $m_{\chi}$, at the representative point: $\tan \beta=20$, $m_{h}=50 \mathrm{GeV}$ and $m_{f}=1.2 m_{x}$. The parameters $M_{2}$ and $\mu$ are varied in the ranges $20 \mathrm{GeV} \leq M_{2} \leq 6 \mathrm{TeV}$ and $20 \mathrm{GeV}$ $\leq|\mu| \leq 3 \mathrm{TeV}$. The horizontal line denotes the present experimental limit $R_{\text {exp }}$. Filled circles represent the $R$ values for neutralino compositions where gaugino and higgsino components are maximally mixed $\left(0.45 \leq a_{1}^{2}+a_{2}^{2} \leq 0.55\right)$.

\footnotetext{
* For an up-to-date review of the present achievements and the new perspectives for dark matter detectors using $\mathrm{Ge}$ as well as the other materials discussed in the present work, see the experimental papers in ref. [20].
}

(at higher $m_{\chi}$ the plots retain the same smooth decrease as $m_{x}$ increases).

To illustrate some of the features of the scatter plot in fig. 1, let us recall two main points: (i) at fixed $m_{x}$ the event rate $R$ depends on the model parameters only through the product $\rho_{\chi} \sigma_{i}$ (see eqs. (2.1), (2.2)), (ii) this product is given by

$$
\begin{array}{r}
\rho_{\chi} \sigma_{i}=\rho_{\ell} \sigma_{i} \text { when } \Omega_{\chi} h^{2} \geq\left(\Omega h^{2}\right)_{\min }, \\
\rho_{\chi} \sigma_{i}=\xi \rho_{\ell} \sigma_{i} \propto\left(\Omega_{\chi} h^{2}\right) \sigma_{i} \propto \frac{\sigma_{i}}{\left\langle\sigma_{\mathrm{ann}} v\right\rangle} \\
\text { when } \Omega_{\chi} h^{2}<\left(\Omega h^{2}\right)_{\min }
\end{array}
$$

( $\sigma_{\text {ann }}$ and $v$ being the $\chi-\chi$ annihilation cross section and the relative velocity; see eq. (3.1) of paper I).

In fig. 1 the wide spread in the values of $R$ at fixed $m_{\chi}$ is due to the very large variations of $\rho_{\chi} \sigma_{i}$ as we scan the $M_{2}, \mu$ plane along an isomass curve. A detailed analysis of our numerical results shows that the maximal value of $R$, at each $m_{\chi}$, usually corresponds to a point in the $M_{2}, \mu$ plane where $\Omega_{x} h^{2}$ is substantially below $\left(\Omega h^{2}\right)_{\min }$. In other words, the maximal expected signal is obtained for neutralino configurations which imply use of eq. (6.2) for $\rho_{\chi}$, with values for the rescaling parameter $\xi$ which may also be very small. The nature of the neutralino composition that at fixed $m_{x}$ provides the maximum $R$ depends on a very crucial balance between $\sigma_{i}$ and $\sigma_{\text {ann }}$ in the expression (6.2). Indicatively, the largest event rates in the plot of fig. 1 entail the following values for $\xi: \xi \sim 0.01-0.02$ for $m_{x} \simeq$ 40-60 GeV (in this case the gaugino-higgsino mixing is substantial), $\xi \sim 0.1$ for $m_{x} \simeq 60-70$ $\mathrm{GeV}$ (here the composition is mainly of the higgsino type), $\xi \sim 0.05$ for $m_{\chi} \simeq 70-90 \mathrm{GeV}$ (large gaugino-higgsino mixing); above $m_{x} \simeq 90 \mathrm{GeV}$ the neutralino composition is mainly of the gaugino type with $\xi$ which reaches values of order of a few tenths. These properties are depicted in figs. 2 and 3. Figure 2 shows $\Omega_{x} h^{2}$ as a function of $m_{x}$; the diamonds denote the values of the relic abundances for those configurations that provide the maximal values of $R$. The compositions of these neutralino configurations are shown in the $M_{2}-\mu$ plane of fig. 3 . 


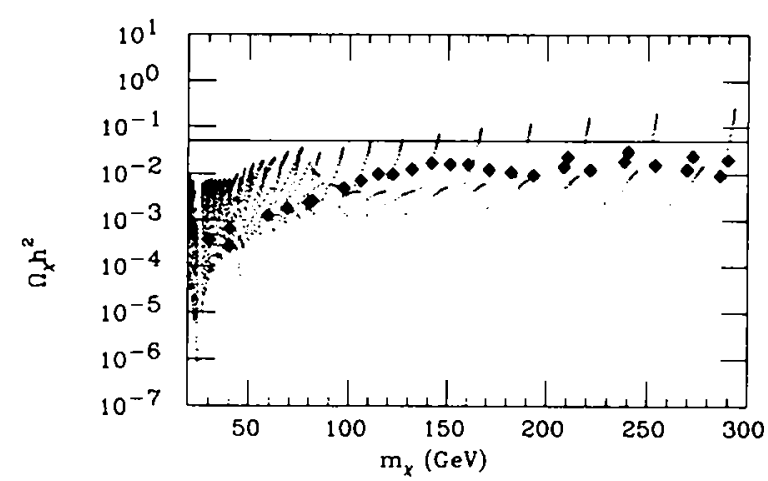

Fig. 2. Scatter plot of the neutralino relic abundance $\Omega_{x} h^{2}$ as a function of neutralino mass $m_{\chi}$. The relic abundance is calculated in the same representative point as in fig. 1 and also with the same variations over $M_{2}, \mu$. The horizontal line denotes the value $\left(\Omega h^{2}\right)_{\min }=0.05$. Diamonds represent the values of relic abundance which give the maximal value of $R$ for a given neutralino mass $m_{x}$ (they correspond to the maxima of $R$ in fig. 1).

In view of these results we wish to emphasize that, by disregarding the neutralino compositions that give low values of $\Omega_{\chi} h^{2}$ (say, below $\Omega h^{2} \sim$ 0.05 ) on the base that these compositions are not cosmologically interesting, one would miss configurations that potentially generate the most sizable signals in the direct searches.

It is worth noticing that the configurations of maximal gaugino-higgsino mixing (defined in paper I) provide large values of $R$ (denoted by circles in fig. 1) over the whole $m_{x}$ range, even if the rescaling effect is always substantial for these compositions; in fact in this case the elastic $\chi^{-}$ nucleus coherent cross sections are very large and compensate for the large values of $\sigma_{\text {ann }}$ in eq. (6.2).

Because of the properties that we have just discussed, the graph of the event rate $R$ (fig. 1) exhibits some of the salient features of the plot of the relic abundance $\Omega_{\chi} h^{2}$ as a function of $m_{x}$ (see fig. 2). In particular we recognize the pronounced dips at $m_{\chi} \sim 25 \mathrm{GeV}$ and at $m_{\chi} \sim 45$ $\mathrm{GeV}$, i.c. at the values where $\sigma_{\text {ann }}$ has an $A(h)-$ pole and a $Z$-pole, respectively.

Finally we wish to stress that in the representative point under discussion the maximal expected signal: $R \sim 6$ events $/ \mathrm{kg}$ day, which occurs at $m_{\chi} \sim 40 \mathrm{GeV}$, differs only by a factor 1.5 from the present experimental sensitivity $R_{\text {exp }} \simeq 10$ events / $\mathrm{kg}$ day [14] (denoted by a horizontal line in our plot of fig. 1). In order to illustrate the discovery potential of the direct neutralino search by $\mathrm{Ge}$ detectors we show in fig. 4 the regions in the $M_{2}, \mu$ plane that could be experimentally investigated in case of improvements in sensitivity by a factor of 10 (heavy-dotted regions) or by a factor 100 (light-dotted regions).

In fig. 5 we give a sample of the differential rates $\mathrm{d} R / \mathrm{d} E_{\mathrm{ec}}$ corresponding to the neutralino compositions that, at a given $m_{\chi}$, provide a maximal value for the integrated rate $R$, previously defined. The values $m_{x}=40,80,150 \mathrm{GeV}$ are
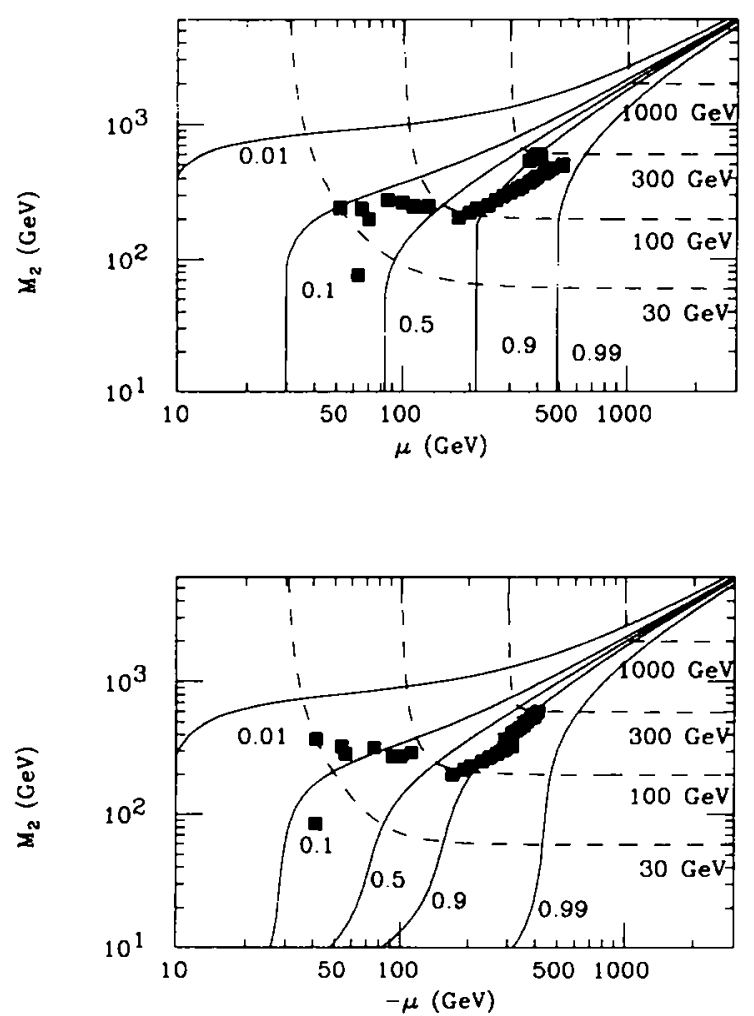

Fig. 3. Isomass curves and composition lines for neutralino in the $M_{2}-\mu$ plane for $\tan \beta=20$. Dashed lines are lines of constant neutralino mass $\left(m_{\chi}=30 \mathrm{GeV}, 100 \mathrm{GeV}, 30\right) \mathrm{GeV}$ and $1 \mathrm{TeV}$ ). Solid lines refer to constant gaugino fraction $f_{g}$ in the neutralino composition $\left(f_{g}=a_{1}^{2}+a_{2}^{2}\right): f_{g}=0.99,0.9$, $0.5,0.1$ and 0.01 . Squares represent the points in the $M_{2}-\mu$ parameter space which give the maximal integrated rate $R$ for a given neutralino mass $m_{\chi}$ (they correspond to the maxima of $R$ in fig. 1). 

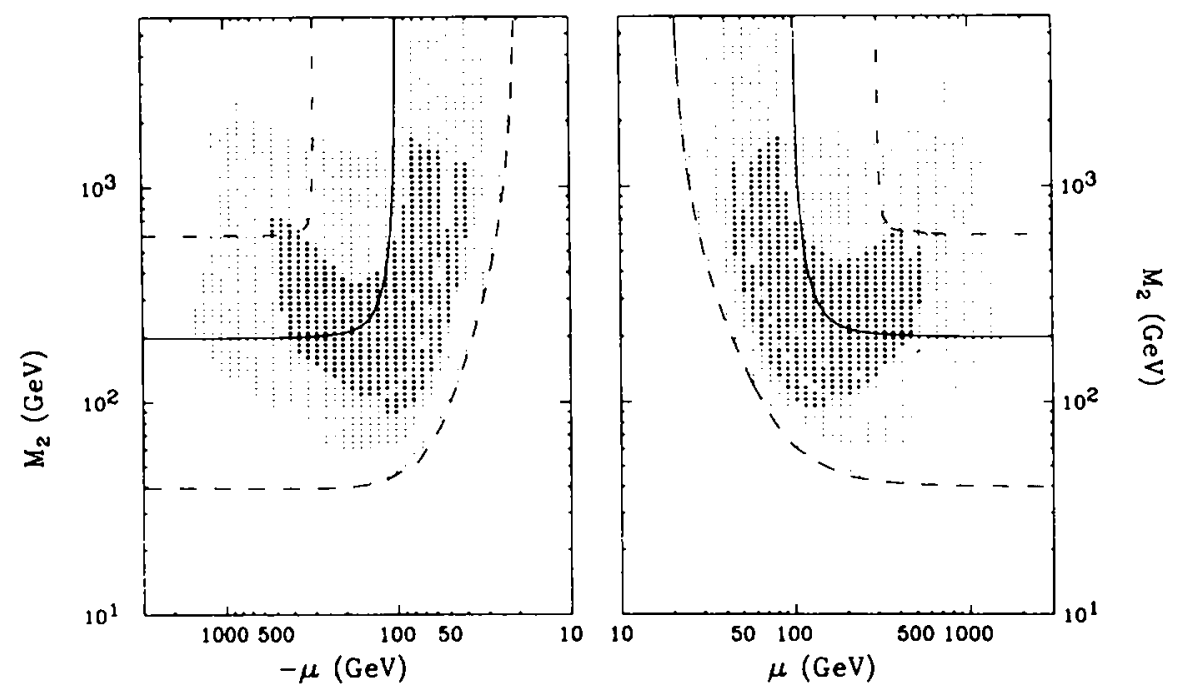

Fig. 4. Explorable regions in the $M_{2}-\mu$ parameter space for neutralino-Ge interaction. Parameters are: $20 \mathrm{GeV} \leq M_{2} \leq 6 \mathrm{TeV}, 20$ $\mathrm{GeV} \leq|\mu| \leq 3 \mathrm{TeV}, \tan \beta=20, m_{h}=50 \mathrm{GeV}$ and $m_{\tilde{f}}=1.2 m_{\chi}$. Neutralino masses extend up to $m_{\chi}=1 \mathrm{TeV}$. Heavy dots denote regions where the integrated rate $R$ is in the range $0.1 R_{\exp }<\leq \leq R_{\text {exp }}$, being $R_{\text {exp }}$ the present experimental bound. In light-dotted regions $0.01 R_{\text {exp }} \leq R \leq 0.1 R_{\exp }$ occurs. Lines denote curves of constant neutralino mass: dashed lines stand for $m_{\chi}=20 \mathrm{GeV}$, solid lines for $m_{\chi}=100 \mathrm{GeV}$, dot-dashed lines for $m_{x}=300 \mathrm{GeV}$ and dotted lines for $m_{\chi}=1 \mathrm{TeV}$.

displayed in the figure together with the present experimental points [14].

In fig. 6 we give the event rate $R$ at the same values of $\tan \beta$ and $m_{h}$ as above, but for the second set of values for the sfermion masses, i.e. $m_{\bar{f}}=3 \mathrm{TeV}$. We notice that in this case the expected maximal rate is even larger than in the previous example for $m_{\chi}>70 \mathrm{GeV}$. This increase

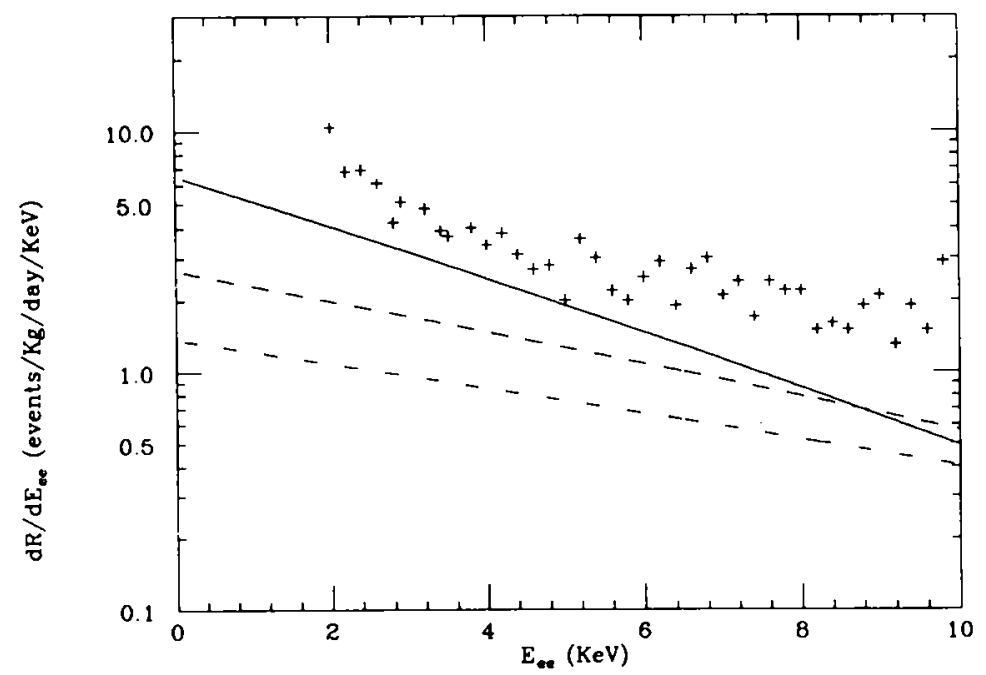

Fig. 5. Nuclear recoil spectrum $\mathrm{d} R / \mathrm{d} E_{\mathrm{cc}}$ for neutralino-Ge interaction as a function of the electron-equivalent energy $E_{\mathrm{cc}}$. Lines denote the nuclear recoil spectra which give the maximal integrated rate $R$ for a fixed neutralino mass: solid line is calculated for $m_{\chi}=40 \mathrm{GeV}$, dashed line for $m_{\chi}=80 \mathrm{GeV}$ and dot-dashed line for $m_{\chi}=150 \mathrm{GeV} . M_{2}$ and $\mu$ parameters are varied in the ranges $20 \mathrm{GeV} \leq M_{2} \leq 6 \mathrm{TeV}$ and $20 \mathrm{GeV} \leq|\mu| \leq 3 \mathrm{TeV}$. The other parameters are: $\tan \beta=20, m_{h}=50 \mathrm{GeV}$ and $m_{f}=1.2 m_{\chi}$. Crosses represent the present experimental limit [14]. 


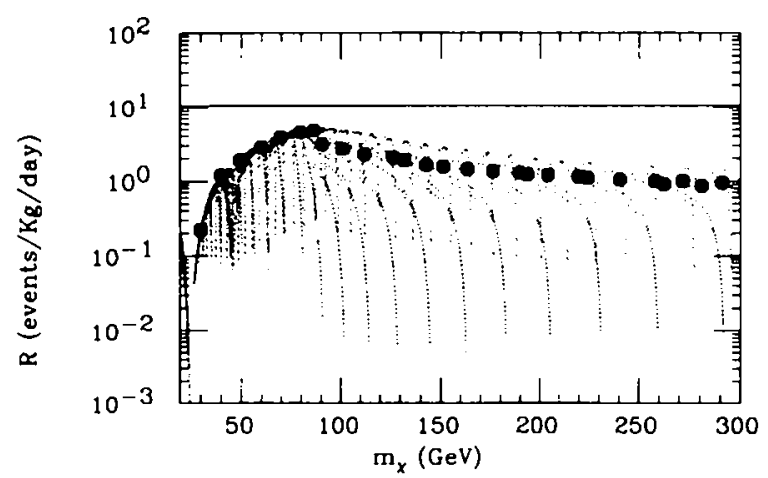

Fig. 6. Same as in fig. 1, except for $m_{f}=3 \mathrm{TeV}$ instead of $m_{\bar{f}}=1.2 m_{x}$.

is due to compositions which are more markedly of the gaugino type (rescaling effect is small here, due to the suppression induced by the high values of $m_{\tilde{f}}$ on the annihilation cross section). The relevant explorable regions in the $M_{2}-\mu$ plane are shown in fig. 7.

Now let us see how the theoretical rates vary as we change the values for $\tan \beta$ and for $m_{h}$. In our evaluations the Higgs-exchange graphs have a substantial role; then, increasing $m_{h}$ has a suppression effect on cross sections, and in particular on the elastic one. This is mainly due to the

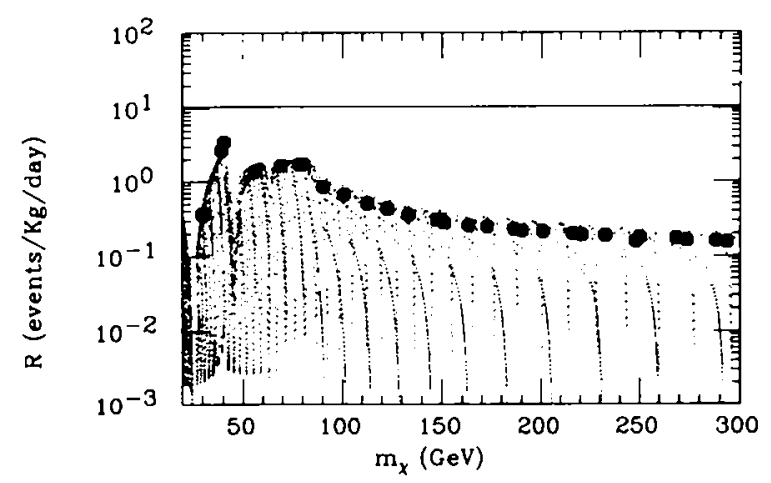

Fig. 8. Same as in fig. 1; here the representative point is: $\tan \beta=8, m_{h}=50 \mathrm{GeV}$ and $m_{\bar{f}}=1.2 m_{\chi}$.

dependence on the Higgs masses through the propagators $\left(\sim 1 / m_{h}^{4}\right)$. $\tan \beta$ enters in some of the coupling constants; it generally occurs that cross sections decrease as $\tan \beta$ decreases. To illustrate the size of these effects, we show in figs. 8 and 9 the integrated event rates for $\tan \beta=8$, $m_{h}=50 \mathrm{GeV}$ for the two sets of values for $m_{\tilde{f}}$ and in figs. 10 and 11 the relevant explorable regions in the $M_{2}, \mu$ plane. In figs. 12 and $13 R$ is displayed at the representative point $\tan \beta=8$, $m_{h}=80 \mathrm{GeV}$ for $m_{\tilde{f}}=1.2 m_{\chi}$ and $m_{\bar{f}}=3 \mathrm{TeV}$
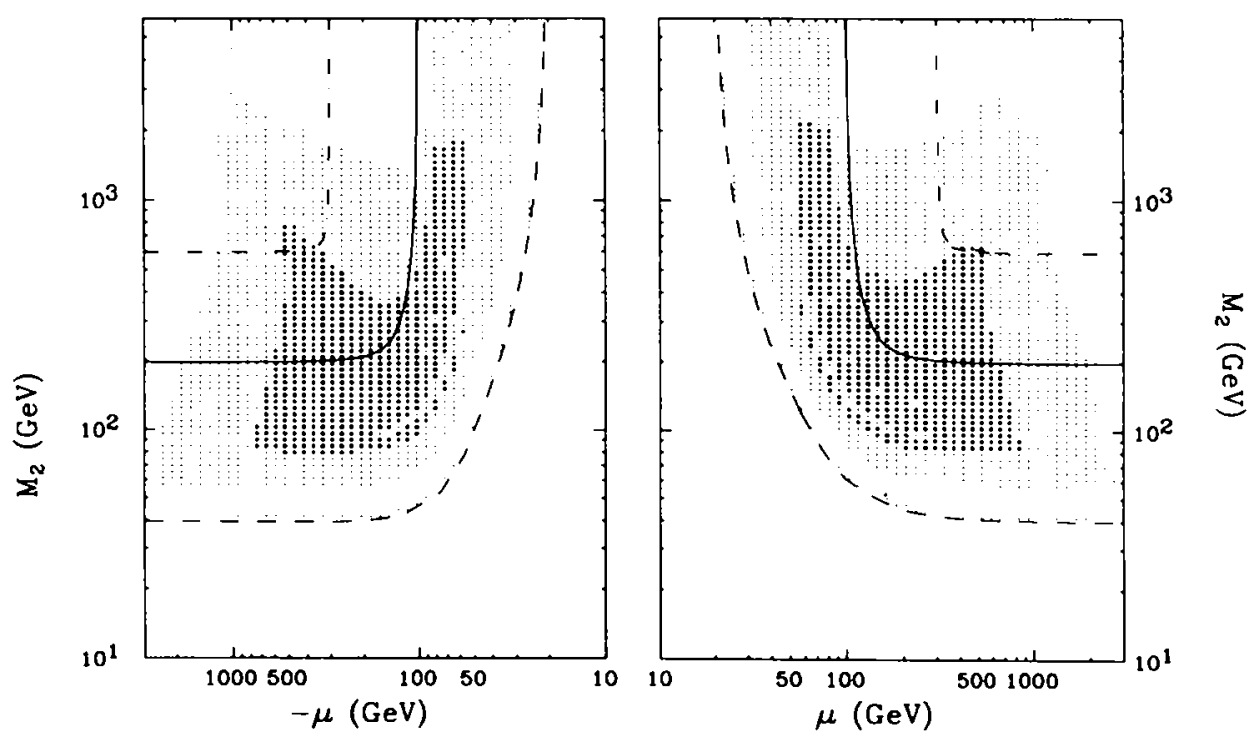

Fig. 7. Same as in fig. 4, except for $m_{j}=3 \mathrm{TeV}$ instead of $m_{j}=1.2 m_{x}$. Dashed line $20 \mathrm{GeV}$; solid line $100 \mathrm{GeV}$; dot-dashed line $300 \mathrm{GeV}$; dotted line $1 \mathrm{TeV}$. 


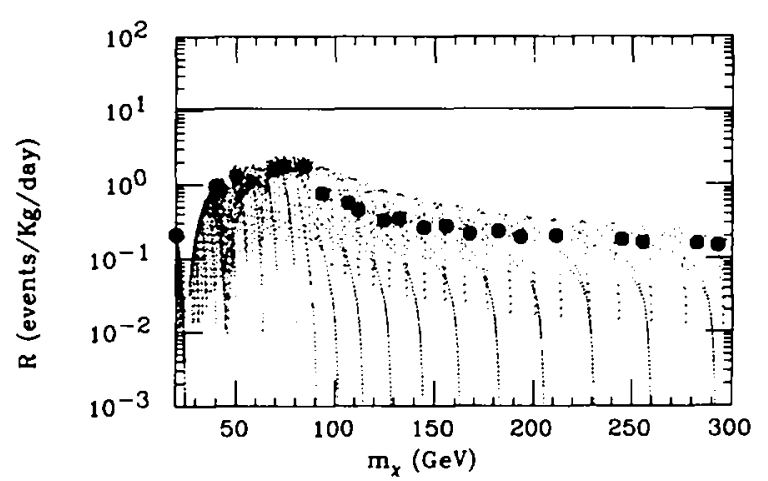

Fig. 9. Same as in fig. 1, here the representative point is: $\tan \beta=8, m_{h}=50 \mathrm{GeV}$ and $m_{f}=3 \mathrm{TeV}$.

respectively. As compared to the previous case $\tan \beta=8, m_{h}=50 \mathrm{GeV}$, the present event rate $R$ is sizably lower, mainly because of the propagator effect $\sim 1 / m_{h}^{4}$. The regions in the $M_{2}, \mu$ plane which are experimentally explorable, by increasing the sensitivity appropriately, are shown in figs. 14 and 15.

We do not report here the results for other representative points. We only quote that for instance at $\tan \beta=2$, as is expected, the explorable regions are smaller than the previous ones. However, a 2-order-of-magnitude improvement in sensitivity would still make feasible an exploration in the range $20 \mathrm{GeV} \leqslant m_{x} \leqslant 100 \mathrm{GeV}$.

Then, as far as the Ge detectors are concerned, we can conclude that improvements of about 2 orders of magnitude in experimental sensitivities would allow investigation of the neutralino dark matter in large regions of the model parameter space. It is extremely encouraging that this level of sensitivities appears to be within reach in the near future [20].

Let us turn now to a brief discussion of the other materials mentioned before. The theoretical analysis has been performed along the same lines as in the case of Ge. For sake of brevity, here we only report the differential and the integrated rates for the two representative points: $\tan \beta=20, m_{h}=50 \mathrm{GeV}, m_{\tilde{f}}=1.2 m_{x}$ and $\tan \beta$ $=20, m_{h}=50 \mathrm{GeV}, m_{f}=3 \mathrm{TeV}$.

\subsection{Sodium iodide}

Theoretical expectations for this diatomic material were already presented and compared with experimental values in ref. [2] for neutralino masses in the range $20 \mathrm{GeV} \leq m_{x} \leq 80 \mathrm{GeV}$.
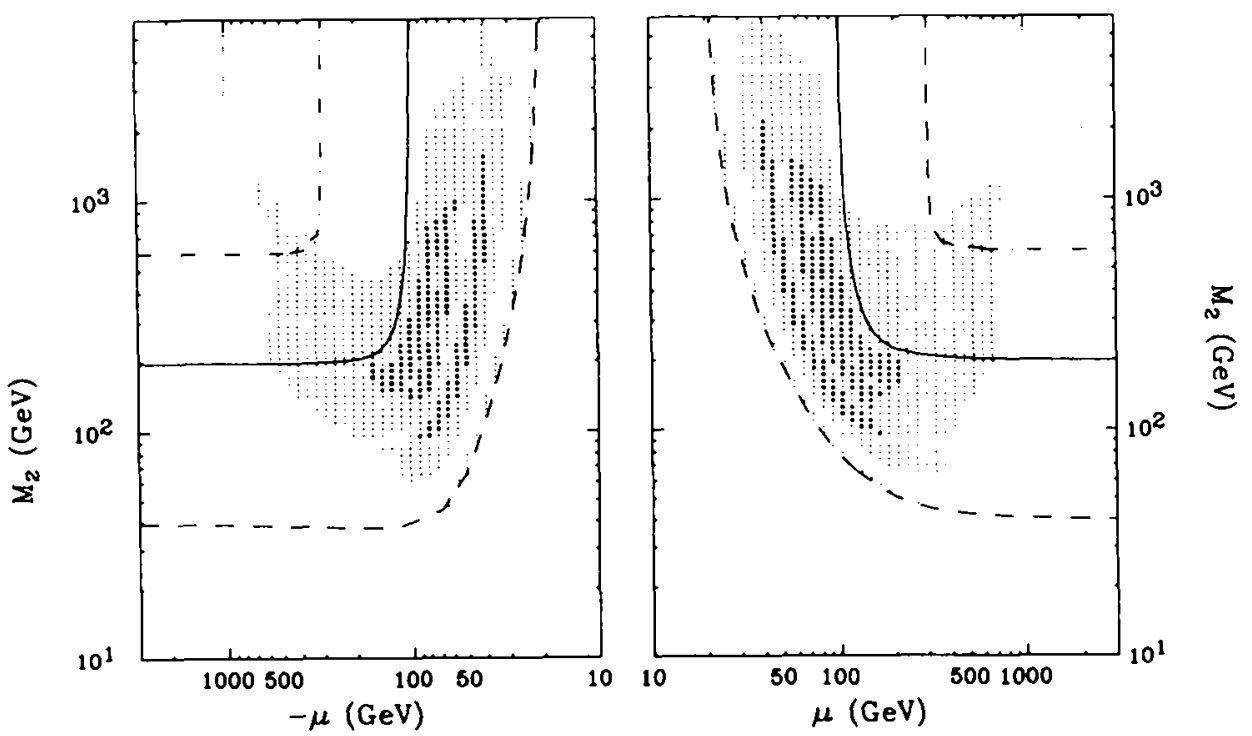

Fig. 10. Same as in fig. 4 , here the representative point is: $\tan \beta=8, m_{h}=50 \mathrm{GeV}$ and $m_{j}=1.2 m_{x}$. Dashed line $20 \mathrm{GeV}$; solid line $100 \mathrm{GeV}$; dot-dashed line $300 \mathrm{GeV}$; dotted line $1 \mathrm{TeV}$. 

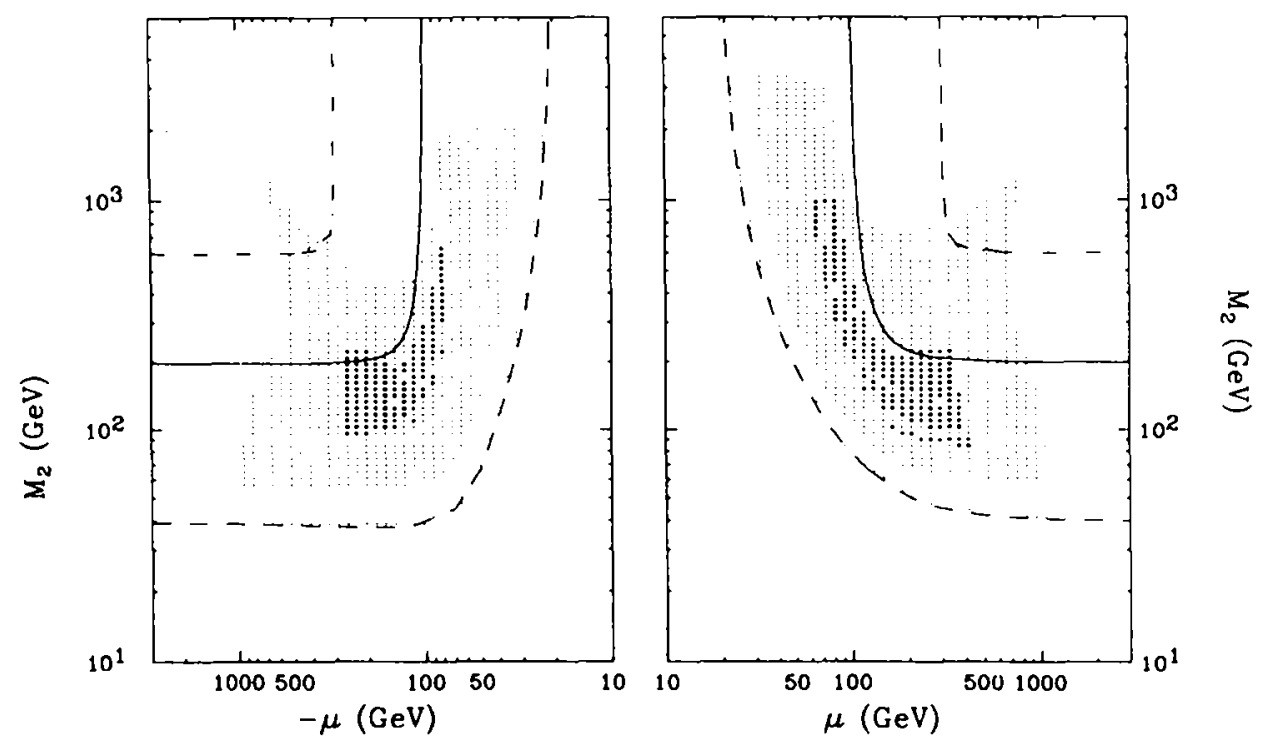

Fig. 11. Same as in fig. 4 , here the representative point is: $\tan \beta=8, m_{h}=50 \mathrm{GeV}$ and $m_{\bar{f}}=3 \mathrm{TeV}$. Dashed line $20 \mathrm{GeV}$; solid line $100 \mathrm{GeV}$; dot-dashed line $300 \mathrm{GeV}$; dotted line $1 \mathrm{TeV}$.

Here the analysis is extended up to $m_{x}=1 \mathrm{TeV}$ and to much wider regions of the parameter space. The results of the integrated rate $R(4.5$ $\left.\mathrm{KeV} \leq E_{\mathrm{ee}} \leq 5 \mathrm{KeV}\right)$ are shown in figs. 16 and 17 .

For the case of $\tan \beta=20, m_{h}=50 \mathrm{GeV}, m_{\tilde{f}}$ $=1.2 m_{x}$ we also give in fig. 18 the maximal differential rate at three values of $m_{x}$. We notice that the peculiar behaviour of $\mathrm{d} R / \mathrm{d} E_{\mathrm{ce}}$ as a function of $m_{x}$ is due to the diatomic nature of $\mathrm{NaI}$. The response of a $\mathrm{NaI}$ detector to the neu-

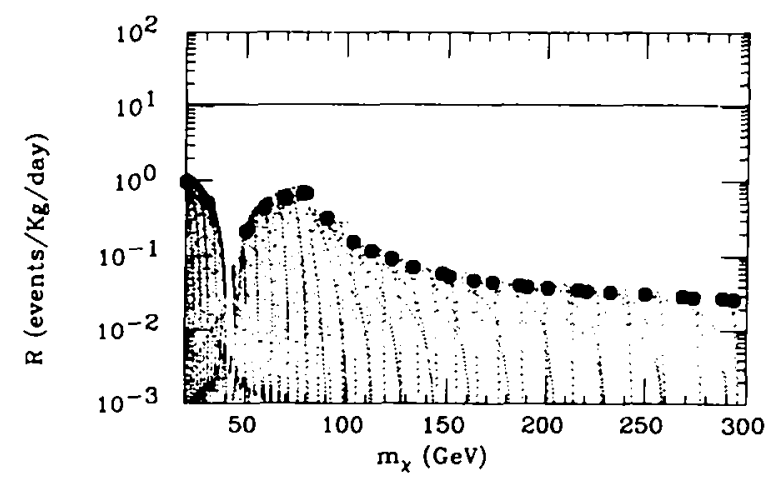

Fig. 12. Same as in fig. 1, here the representative point is: $\tan \beta=8, m_{h}=80 \mathrm{GeV}$ and $m_{f}=1.2 m_{\chi}$. tralino scattering is dominated by iodine up to $E_{\mathrm{ce}} \sim 6 \mathrm{keV}$ and by Sodium at higher $E_{\mathrm{ee}}$. This circumstance depends on the very different coherent form factor effects in the two nuclei as is shown by the values reported in table 1 . From the threshold up to $E_{\mathrm{ee}} \sim 6 \mathrm{KeV}$ in iodine the large suppression due to $F^{2}\left(E_{\mathrm{R}}^{\mathrm{th}}\right)$ is compensated by a large value of its coherent pointlike cross section ( $A^{2}$ effect). At higher values of $E_{\text {ec }}$ the iodine coherent form factor has a fast fall-off, because

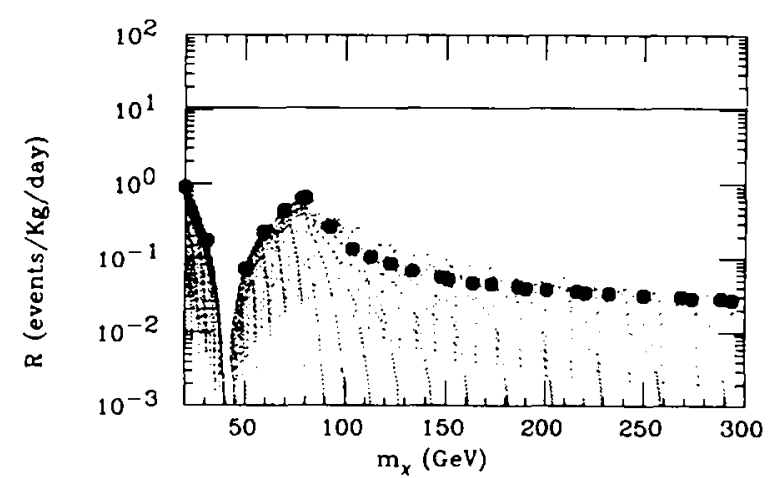

Fig. 13. Same as in fig. 1, here the representative point is: $\tan \beta=8, m_{h}=80 \mathrm{GeV}$ and $m_{f}=3 \mathrm{TeV}$. 

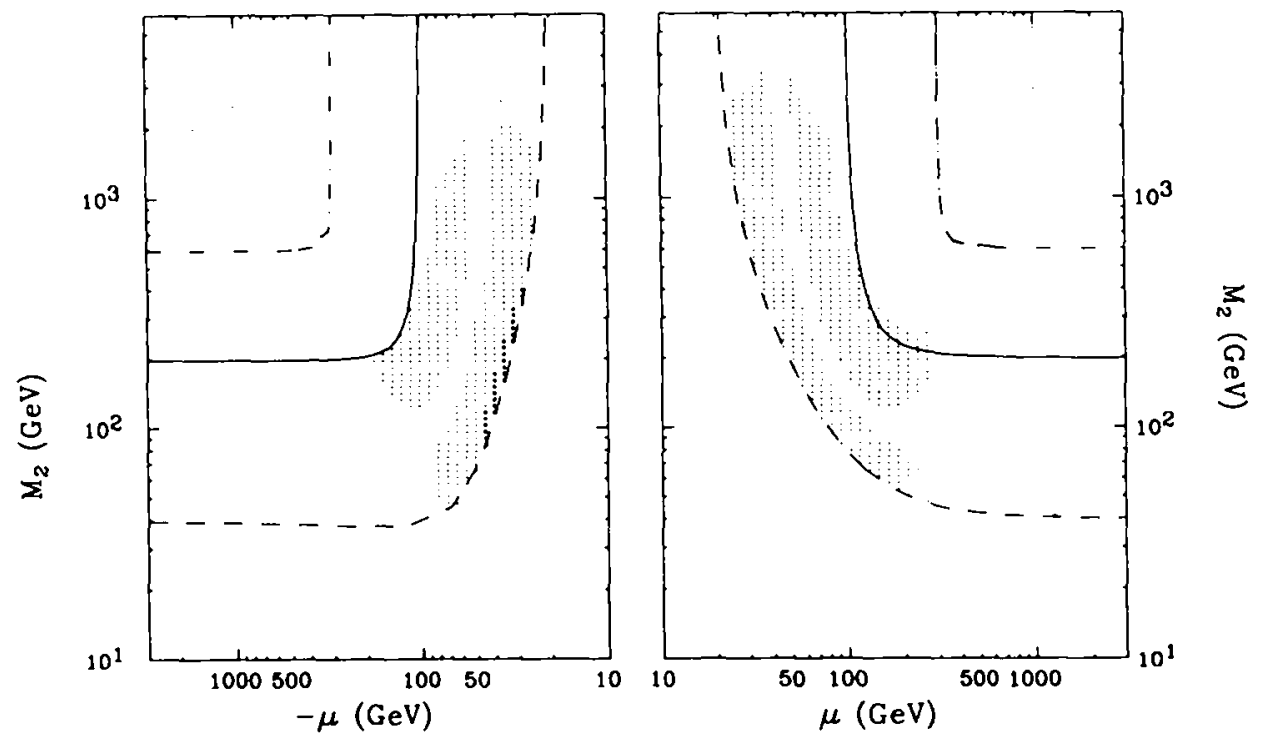

Fig. 14. Same as in fig. 4 , here the representative point is: $\tan \beta=8, m_{h}=80 \mathrm{GeV}$ and $m_{f}=1.2 m_{\chi}$. Dashed line $20 \mathrm{GeV}$; solid line $100 \mathrm{GeV}$; dot-dashed line $300 \mathrm{GeV}$; dotted line $1 \mathrm{TeV}$.

of the zero at $E_{\mathrm{ee}}=7.4 \mathrm{keV}$, whereas in the whole range of $E_{\mathrm{ee}}$ shown in fig. 18 the sodium form factor has a rather flat behaviour.
As discussed in ref. [2] an improvement of two orders of magnitude in the NaI detectors sensitivity is foreseeable in the next few years. This
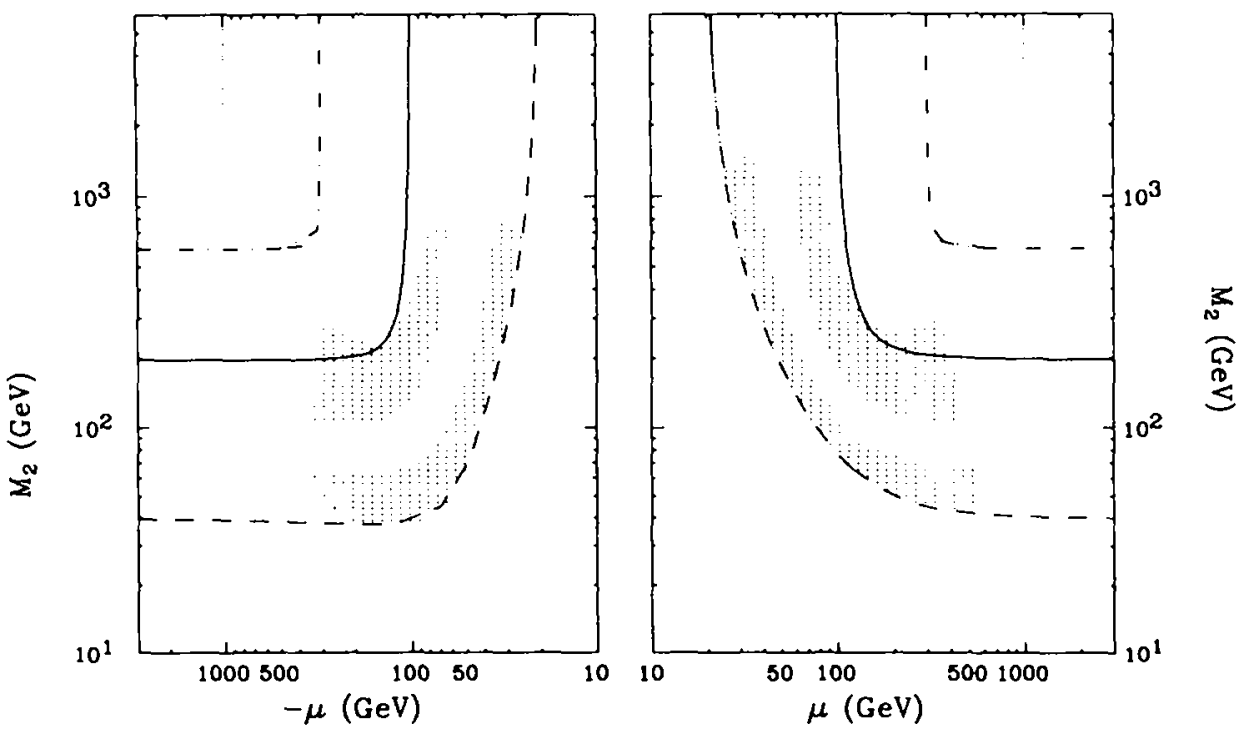

Fig. 15. Same as in fig. 4 , here the representative point is: $\tan \beta=8, m_{h}=80 \mathrm{GeV}$ and $m_{f}=3 \mathrm{TeV}$. Dashed line $20 \mathrm{GeV}$; solid line $100 \mathrm{GeV}$; dot-dashed line $300 \mathrm{GeV}$; dotted line $1 \mathrm{TeV}$. 


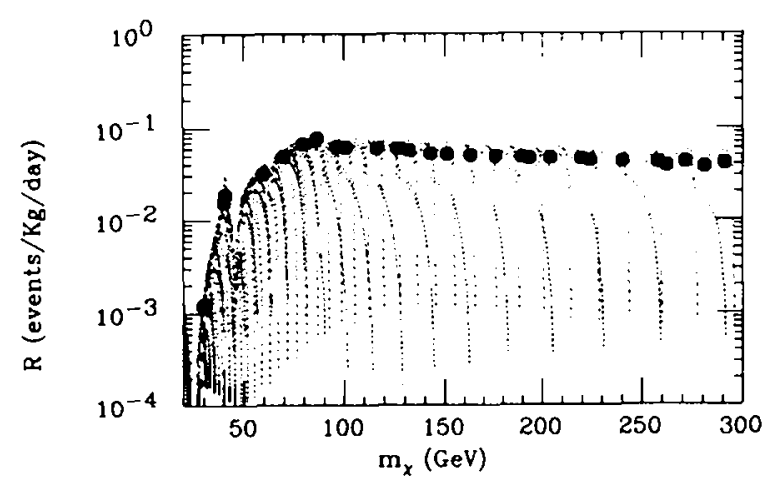

Fig. 16. Scatter plot of the integrated rate $R\left(4.5 \mathrm{keV} \leq E_{\mathrm{ce}} \leq 5\right.$ $\mathrm{keV}$ ) for neutralino- $\mathrm{NaI}$ interaction. The representative point is: $\tan \beta=20, m_{h}=50 \mathrm{GeV}$ and $m_{f}=1.2 \mathrm{~m}_{\chi}$; the parameters $M_{2}$ and $\mu$ are varied in the ranges $20 \mathrm{GeV} \leq M_{2} \leq 6 \mathrm{TeV}$ and $20 \mathrm{GeV} \leq|\mu| \leq 3 \mathrm{TeV}$. Filled-circles represent the $R$ values for neutralino compositions where gaugino and higgsino components are maximally mixed $\left(0.45 \leq a_{1}^{2}+a_{2}^{2} \leq 0.55\right)$.

would allow to start the exploration of the physics of dark matter neutralinos with these detectors.

\subsection{Xenon}

Much experimental activity is now being developed in dark matter detectors which employ Xe.

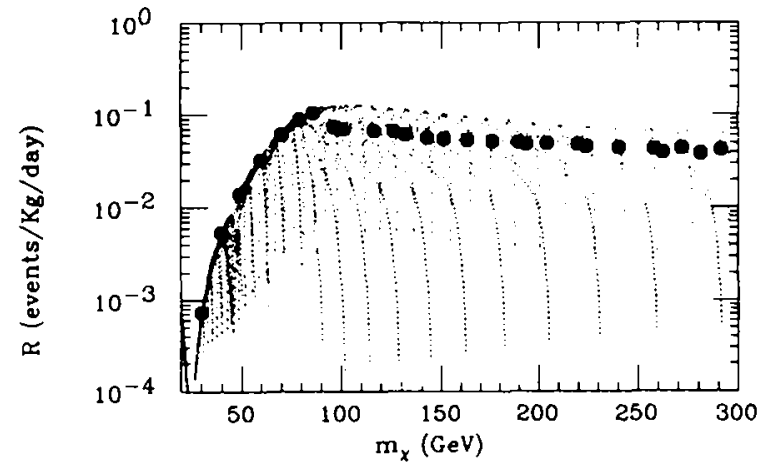

Fig. 17. Same as in fig. 16, except for $m_{f}=3 \mathrm{TeV}$ instead of $m_{j}=1.2 m_{x}$.

We report in figs. 19 and 20 the integrated rate $R\left(20 \mathrm{keV} \leq E_{\mathrm{cc}} \leq 22 \mathrm{keV}\right)$ in the representative points: $\tan \beta=20, m_{h}=50 \mathrm{GeV}, m_{f}=1.2 m_{\chi}$ and $\tan \beta=20, m_{h}=50 \mathrm{GeV}, m_{f}=3 \mathrm{TeV}$. For the first of these points also the differential rate is given (fig. 21). We notice that for $\mathrm{Xe}$ the suppression due to the coherent form factor is sizeable (see table 1). However the use of Xe for detecting dark matter neutralinos appear to be

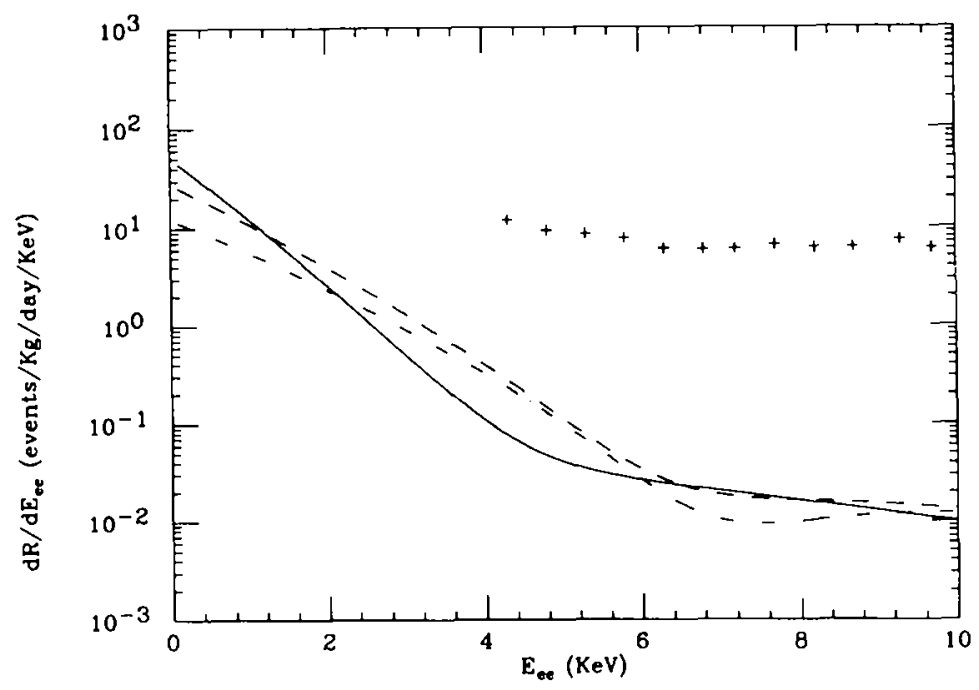

Fig. 18. Nuclear recoil spectrum $\mathrm{d} R / \mathrm{d} E_{\mathrm{ec}}$ for neutralino-NaI interaction as a function of the electron-equivalent energy $E_{c e}$. Lines denote the nuclear recoil spectra which give the maximal integrated rate for a fixed neutralino mass: solid line is calculated for $m_{x}=40 \mathrm{GeV}$, dashed line for $m_{\chi}=80 \mathrm{GeV}$ and dot-dashed line for $m_{x}=150 \mathrm{GeV}$. $M_{2}$ and $\mu$ parameters are varied in the ranges $20 \mathrm{GeV} \leq M_{2} \leq 6 \mathrm{TeV}$ and $20 \mathrm{GeV} \leq|\mu| \leq 3 \mathrm{TeV}$. The other parameters are: $\tan \beta=20, m_{h}=50 \mathrm{GeV}$ and $m_{f}=1.2 m_{\chi}$. Crosses represent the present experimental limit [16]. 


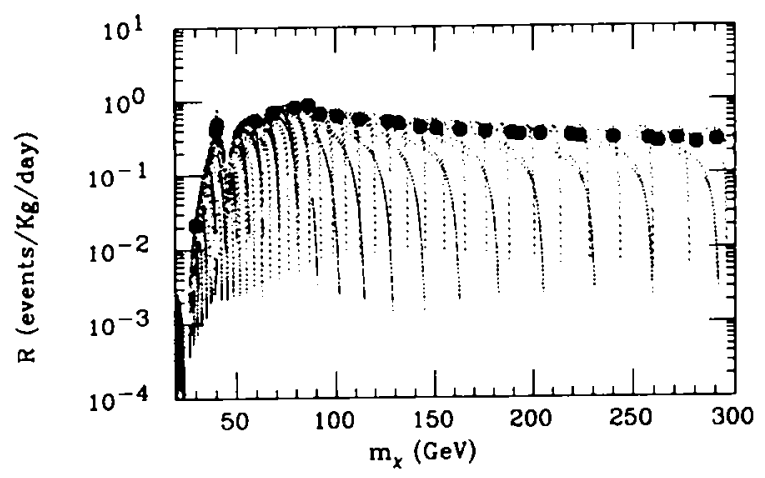

Fig. 19. Scatter plot of the integrated rate $R\left(20 \mathrm{keV} \leq E_{\mathrm{ec}} \leq 22\right.$ $\mathrm{keV}$ ) for neutralino-Xe interaction. The values for the model parameters and the notations are as in fig. 16.

very promising in view of the experimental features of these detectors.

\subsection{Calcium fluoride}

Finally in figs. 22 and 23 we display the integrated rate $R\left(8.5 \mathrm{keV} \leq E_{\mathrm{ce}} \leq 9 \mathrm{keV}\right)$ for $\mathrm{CaF}_{2}$ in the usual representative points: $\tan \beta=20$, $m_{h}=50 \mathrm{GeV}, m_{\tilde{f}}=1.2 m_{\chi}$ and $\tan \beta=20, m_{h}$ $=50 \mathrm{GeV}, m_{f}=3 \mathrm{TeV}$. In fig. 24 also the differ-

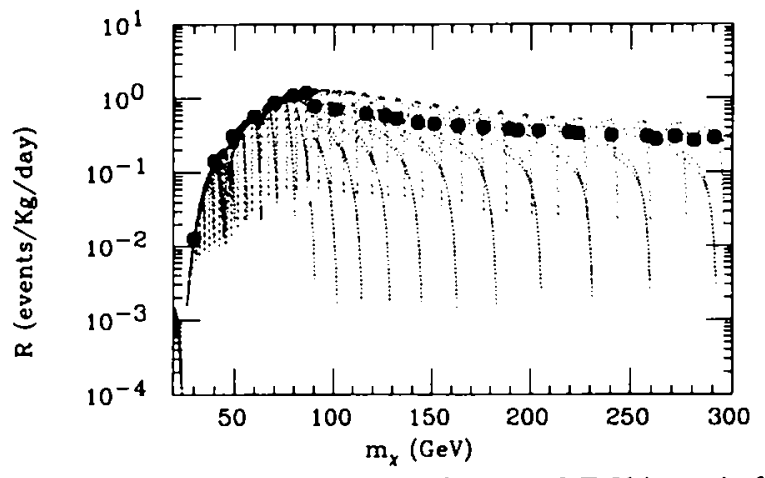

Fig. 20. Same as in fig. 19, except for $m_{f}=3 \mathrm{TeV}$ instead of $m_{f}=1.2 m_{x}$

ential rate for the first representative point is shown.

\section{Conclusions}

We can conclude that the use of different materials and the achievements of improved experimental performances are offering very exciting perspectives for a direct search of neutralino as a dark matter component.

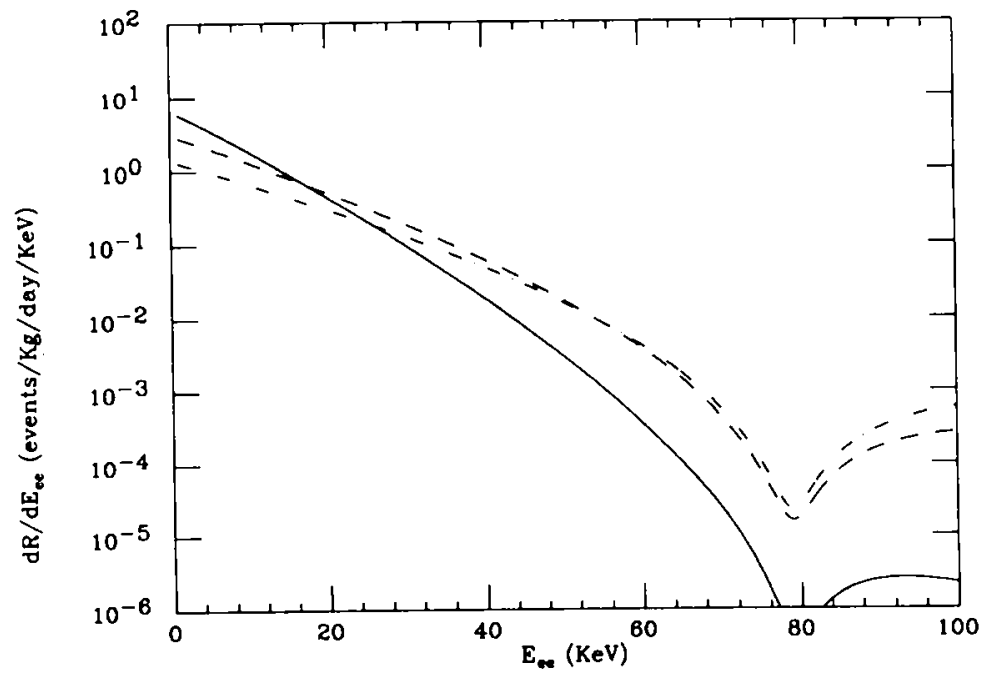

Fig. 21. Nuclear recoil spectrum $\mathrm{d} R / \mathrm{d} E_{\mathrm{ee}}$ for neutralino-Xe interaction as a function of the electron-equivalent energy $E_{\mathrm{ce}}$. The values for the model parameters and the notations are as in fig. 18. Here no experimental data are available. 


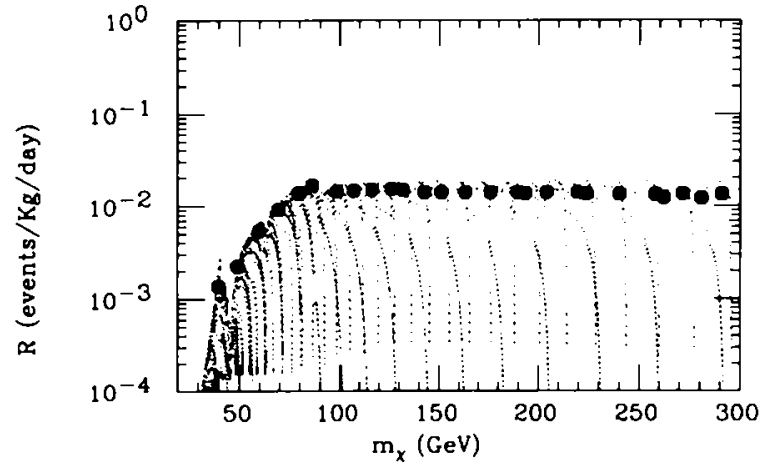

Fig. 22. Scatter plot of the integrated rate $R\left(8.5 \mathrm{keV} \leq E_{\mathrm{cec}} \leq 9\right.$ $\mathrm{keV})$ for neutralino- $\mathrm{CaF}_{2}$ interaction. The values for the model parameters and the notations are as in fig. 16 .

The theoretical evaluations presented here aim at setting the level of the experimental sensitivities required to start the exploration of the physics of dark matter neutralinos. The quantitative relations between the experimental sensitivity of a particular detector and its discovery potential are easily readable in our plots.

Some final comments on the main theoretical uncertainties are in order here:

(i) major improvements in theoretical predictive power require some experimental informa-

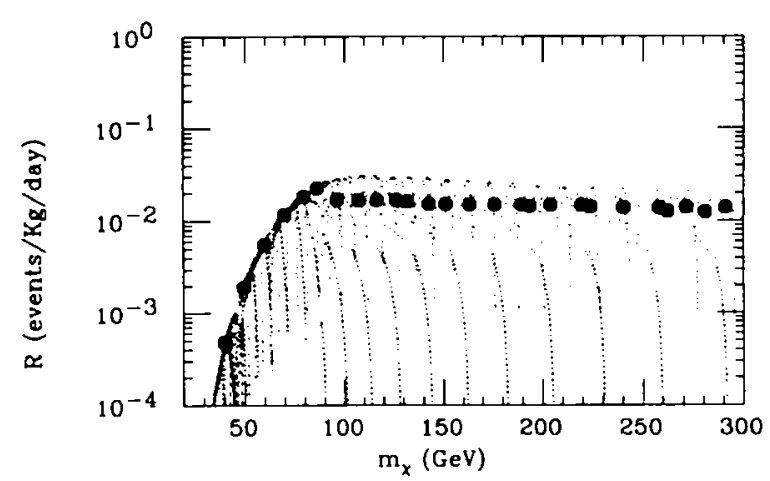

Fig. 23. Same as in fig. 22, except for $m_{j}=3 \mathrm{TeV}$ instead of $m_{j}=1.2 m_{\chi}$.

tions about the masses of particles to be discovered at accelerators: Higgs bosons, SuSy particles;

(ii) the evaluation of the elastic coherent cross section suffers from an uncertainty in the estimate of the Higgs-nucleon strength. In ref. [3] we have analyzed the relevance of this effect for the direct neutralino search, by comparing different estimates for the Higgs-nucleon strength $[21,22]$. A new recent study of this fundamental coupling has shown that the problem needs further investigation [23]. In the present paper we have em-

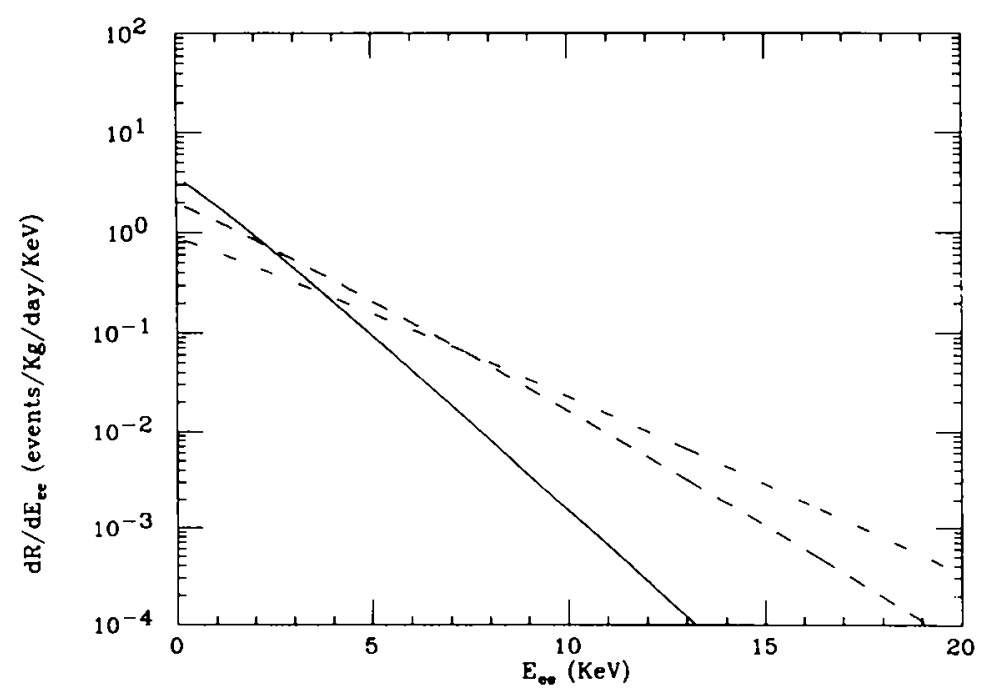

Fig. 24. Nuclear recoil spectrum $\mathrm{d} R / \mathrm{d} E_{\mathrm{ec}}$ for neutralino- $\mathrm{CaF}_{2}$ interaction as a function of the electron-equivalent energy $E_{\mathrm{cec}}$. The values for the model parameters and the notations are as in fig. 18. Here no experimental data are available. 
ployed for the Higgs-nucleon coupling the estimate of ref. [21]. Use of the estimate in ref. [22] would reduce our rates by a factor of 3 approximately;

(iii) the value of $\left(\Omega h^{2}\right)_{\min }$ to be employed for rescaling the local neutralino density is obviously somewhat arbitrary. In the present paper we have conservatively taken $\left(\Omega h^{2}\right)_{\min }=0.05$. In view of the small value of $\Omega$ which appears to be implied by the estimated amount of dark matter in our galaxy, our values for $\left(\Omega h^{2}\right)_{\min }$ could quite well be reduced by a factor of 2 . This reduction in $\left(\Omega h^{2}\right)_{\min }$ would increase the maximal rate $R$ by a factor of 2 , since, as we have seen above, maximal signals occur in general for neutralino compositions where the rescaling effect is large.

\section{Acknowledgement}

This work was supported in part by Research Funds of the Ministero dell'Università e della Ricerca Scientifica e Technologica.

\section{References}

[1] A. Bottino, V. de Alfaro, N. Fornengo, G. Mignola and M. Pignone, Astroparticle Phys. 2 (1994) 67.

[2] A. Bottino, V. de Alfaro, N. Fornengo, G. Mignola, S. Scopel and C. Bacci et al. (BRS Collaboration), Phys. Lett. B 295 (1992) 330.

[3] A. Bottino, V. de Alfaro, N. Fornengo, A. Morales, J. Puimedon and S. Scopel, Mod. Phys. Lett. A 7 (1992) 733.

[4] R. Barbieri, M. Frigeni and G.F. Giudice, Nucl. Phys. B 313 (1989) 725.
[5] K. Griest, Phys. Rev. D 38 (1988) 2357; Phys. Rev. Lett. 61 (1988) 666.

[6] L.G. Pondrom (CDF Collaboration), presented at XXV Int. Conf. on High Energy Physics, Singapore 1990, published in Singapore HEP (1990) 144.

[7] F. Abe et al. (CDF Collaboration), Phys. Rev. Lett. 69 (1992) 3439.

[8] H. Baer, X. Tata and J. Woodside, Phys. Rev. D 44 (1991) 207.

[9] D. Decamp et al. (ALEPH Coll.), Phys. Rep. 216 (1992) 253.

[10] J. Ellis and R. Flores, Phys. Lett. B 263 (1991) 259; Phys. Lett. B 300 (1993) 175.

[11] J. Engel and P. Vogel, Phys. Rev. D 40 (1989) 3132; A.F. Pacheco and D.D. Strottman, Phys. Rev. D 40 (1989) 2131;

F. Iachello, L.M. Krauss and G. Maino, Phys. Lett. B 254 (1991) 220.

[12] J. Engel, Phys. Lett. B 264 (1991) 114.

[13] P. Belli, R. Bernabei and G. Gerbier, private communications.

[14] D. Reusser et al., Phys. Lett. B 255 (1991) 143.

[15] D.O. Caldwell et al., Phys. Rev. Lett. 61 (1988) 510; S.P. Ahlen et al., Phys. Lett. B 195 (1987) 603; E. García et al., Nucl. Phys. B (Proc. Suppl.) 28A (1992) 286.

[16] P. Belli et al., Phys. Lett. B 293 (1992) 460.

[17] P. Belli et al., LNGS-93/55 May 1993, preprint; P. Belli et al., Nucl. Instr. Meth. Phys. Res. A 316 (1992) 55.

[18] J. Engel, S. Pittel, E. Ormand and P. Vogel, Phys. Lett. B 275 (1992) 119.

[19] M.T. Ressel, M.B. Auferheide, S.D. Bloom, K. Griest, G.J. Mathews and D.A. Resler, preprint UCRL-JC114085, 1993.

[20] Proceedings of the Workshop on "The Dark Side of the Universe", Rome, June 1993 (World Scientific, Singapore) to be published.

[21] T.P. Cheng, Phys. Rev. D 38 (1988) 2869; H.-Y. Cheng, Phys. Lett. B 219 (1989) 347.

[22] J. Gasser, H. Leutwyler and M.E. Sainio, Phys. Lett. B 253 (1991) 252.

[23] M. Drees and M.M. Nojiri, Phys. Rev. D 47 (1993) 4226. 